Research article

\title{
High trait anxiety is associated with attenuated feedback-related negativity in risky decision making
}

\author{
Ádám Takács ${ }^{\mathrm{a}, *}$, Andrea Kóbor $^{\mathrm{b}}$, Karolina Janacsek ${ }^{\mathrm{a}, \mathrm{c}}$, Ferenc Honbolygó $^{\mathrm{a}, \mathrm{b}}$, \\ Valéria Csépe ${ }^{\mathrm{b}, \mathrm{d}}$, Dezső Németh ${ }^{\mathrm{a}, \mathrm{c}}$ \\ a Institute of Psychology, Eötvös Loránd University, Izabella utca 46., H-1064, Budapest, Hungary \\ ${ }^{\mathrm{b}}$ Brain Imaging Centre, Research Centre for Natural Sciences, Hungarian Academy of Sciences, Magyar tudósok körútja 2., H-1117, Budapest, Hungary \\ ${ }^{\mathrm{c}}$ MTA-ELTE NAP B Brain, Memory and Language Research Group, Institute of Cognitive Neuroscience and Psychology, Research Centre for Natural Sciences, \\ Hungarian Academy of Sciences, Magyar tudósok körútja 2., H-1117, Budapest, Hungary \\ ${ }^{\mathrm{d}}$ Department of Cognitive Science, Budapest University of Technology and Economics, Egry József utca 1., H-1111, Budapest, Hungary
}

\section{H I G H L I G H T S}

- High (HTA) and low trait anxiety (LTA) adults performed a risk-taking task.

- ERPs were measured in the balloon analogue risk task.

- HTA adults showed smaller FRN after negative feedback than LTA adults.

- The findings support the pessimistic expectation bias in anxiety.

\section{A R T I C L E I N F O}

\section{Article history:}

Received 22 September 2014

Received in revised form 29 May 2015

Accepted 11 June 2015

Available online 17 June 2015

\section{Keywords:}

Anxiety

Balloon analogue risk task

Event-related brain potentials (ERPs)

Feedback-related negativity

Risk-taking behavior

\begin{abstract}
A B S T R A C T
Expectation biases could affect decision making in trait anxiety. Studying the alterations of feedback processing in real-life risk-taking tasks could reveal the presence of expectation biases at the neural level. A functional relevance of the feedback-related negativity (FRN) is the expression of outcome expectation errors. The aim of the study was to investigate whether nonclinical adults with high trait anxiety show smaller FRN for negative feedback than those with low trait anxiety. Participants $(N=26)$ were assigned to low and high trait anxiety groups by a median split on the state-trait anxiety inventory trait score. They performed a balloon analogue risk task (BART) where they pumped a balloon on a screen. Each pump yielded either a reward or a balloon pop. If the balloon popped, the accumulated reward was lost. Participants were matched on their behavioral performance. We measured event-related brain potentials time-locked to the presentation of the feedback (balloon increase or pop). Our results showed that the FRN for balloon pops was decreased in the high anxiety group compared to the low anxiety group. We propose that pessimistic expectations triggered by the ambiguity in the BART decreased outcome expectation errors in the high anxiety group indicated by the smaller FRN. Our results highlight the importance of expectation biases at the neural level of decision making in anxiety.
\end{abstract}

(c) 2015 Elsevier Ireland Ltd. All rights reserved.

\section{Introduction}

Anxiety is an emotional and motivational state characterized by negative outcome expectations and concerns about potential threats [1,2]. Neuroimaging and electrophysiological studies suggest that in high trait anxiety (HTA) and related psychi-

\footnotetext{
* Corresponding author.

E-mail address: adamtakacs@caesar.elte.hu (Á. Takács).
}

atric syndromes, such as obsessive-compulsive disorder (OCD) or generalized anxiety disorder (GAD), performance monitoring is altered [3-5]. Altered performance monitoring could lead to difficulties in everyday risky decision making, often reported in anxiety related syndromes [6-8]. One of the psychophysiological correlates of performance monitoring is the feedback-related negativity (FRN), which has been smaller in HTA than in LTA for negative outcomes [2,9]. In this study, we compared the FRN between HTA and LTA individuals in a task measuring risky decision making. 
The FRN is a frontocentral negative deflection that peaks 200-300 ms after the onset of a negative feedback stimuli [10-12]. As the reinforcement learning theory suggests, the FRN reflects a prediction error of the actual outcome [13]. The FRN most likely related to the functional interaction between the posterior medial frontal cortex and the lateral prefrontal cortex [14]. The FRN is usually followed by a P3 indicating the elaboration of feedback [15].

A study investigated the FRN in trait anxiety in a simple monetary gambling task [2]. Participants chose between two alternatives presented on a screen and each choice was followed by one of three possible outcomes: reward (positive), loss (negative), and ambiguous outcome. The FRN after negative feedback was smaller in HTA adults than in LTA adults. This difference was replicated in a followup experiment that introduced a fourth, neutral condition, as well [9]. The authors suggested that a so-called pessimistic expectation bias caused the difference in FRN between LTA and HTA participants [2,9]. This bias implies that HTA adults have a tendency to negatively interpret any ambiguous stimulus or to expect negative outcomes in gambling situations [2]. Since HTA adults expect more feedback to be negative, they face with less expectation error, which could lead to smaller FRN.

During risky decisions, anxiety can determine the individual's behavior if the negative and positive valence systems, such as fear and reward processing, have equivalent activation [16]. Situations with a high level of perceived risk or ambiguity can trigger these concurrent positive and negative activations. Previous anxiety studies investigated the effect of ambiguous feedback in gambling tasks [2,9]. However, simple choices in these tasks represent only one side of risk-taking behavior [17]. From an economical point of view, risk is the amount of outcome variability. In contrast, nonprofessionals perceive risk as being exposed to potential loss. Moreover, risk-taking behavior usually consists of a series of choices before any negative feedback can possibly occur, such as drinking one more dose of alcohol or increasing bet in a game. Thus in this study, participants performed a task which reflects both of the above-mentioned risk concepts and which is also comparable to real-world risk-taking [17].

To understand ambiguous decision making and real-world risk taking, the balloon analogue risk task (BART) is a widely used experimental tool [17-19]. In this gambling paradigm, participants are asked to pump a balloon on a screen, and each pump is associated either with a reward (positive feedback) or with a balloon burst (negative feedback). After each pump, participants could decide to collect the accumulated reward or to take the risk and pump the balloon further. If the balloon burst, the reward is lost. As a general rule, each successful pump increases the probability of a burst in the next trial; however, this regularity is not transparent for the participants [18]. Participants make a series of choices with increasing risk before they face an external punishment or change their course of action and choose the safe option [17]. Therefore, without explicitly knowing the probabilities of rewards and losses, BART performance requires ambiguous decision making [18,20].

Individual differences can modulate the FRN amplitude in the BART. Namely, it is smaller in the presence of risk for developing alcoholism [21], and it is higher in high executive functions (EFs) [22]. Our previous study extended the findings of Fein and Chang [21] by showing that healthy individuals with high level of EFs might use a model-based strategy in this task, indicated by the enhanced FRN amplitude [22]. However, to the best of our knowledge, there is no available result on the relationship between trait anxiety and FRN in the BART.

In this study, we measured event-related brain potentials (ERPs) in the BART with HTA and LTA adults to understand the neurophysiological underpinnings of risky decision making in anxiety involving a series of choices. We expected smaller FRN in the HTA than in the LTA group, in line with previous findings [2,9].

\section{Materials and method}

\subsection{Participants}

Thirty-nine undergraduate students were recruited to the experiment. Seven participants were excluded by reason of presenting excessive artifacts or not having enough negative-feedback locked epochs for the analysis (see 2.3., EEG measurement and analysis). Participants were divided into LTA and HTA groups by median split of the state-trait anxiety inventory (STAI) - Trait Version Tscore $[23,24]$ (see Fig. 1A). It is a widely used tool to measure trait anxiety with good psychometrical properties [24]. Two participants above the clinical cut-off score (16) on the BDI [25] were excluded to control the clinical level of depression. LTA and HTA groups were matched on the mean adjusted number of pumps (with a maximum difference of one pump, see Section 2.2. Experimental task) in order to account for main behavioral between-group differences in the BART, such as the number of pops and total score (see Table 1). In this way, potential ERP differences cannot be interpreted as a difference in behavioral strategies or as different event frequencies in the task. Four of the participants were excluded by this reason. Thus, the final sample consisted of thirty participants assigned to LTA $(n=13)$ and HTA $(n=13)$ groups. This is a restricted sample of our previous analysis [22], and we only report non-overlapping results specific to the effects of anxiety. Table 1 summarizes questionnaire and behavioral data.

All participants had normal or corrected-to-normal vision, reported normal hearing, and a lack of psychiatric or neurological history. The LTA group consisted of 3 males and 10 females, all of them right-handed; while the HTA group consisted of females only, including 4 left-handed and 9 right-handed adults. Before the laboratory assessment, participants completed four personality questionnaires online: the barratt impulsiveness scale [27,28], the brief sensation seeking scale (BSSS) [29,30], the beck depression inventory (BDI) short version [25,31], and the state-trait anxiety inventory $[23,24]$. Before the assessment, participants received a description of the study and they gave their informed consent. The procedures were approved by the Institutional Review Board of Eötvös Loránd University, Hungary. Participants received course credit for taking part in the ERP experiment.

\subsection{Experimental task}

In the laboratory, participants performed an EEG adapted version of the BART [21,22]. The general structure and visual features of the task was the same as in a previous study [22]. Participants were asked to pump a balloon by pressing one of the response keys. They were instructed to collect as many points as possible by balloon pumps. Participants could see the instruction and task description on the screen as long as they needed.

The pump could have led to an increase in the size of the balloon together with an increase in the reward score presented inside it (positive feedback) or to a balloon burst (negative feedback). Instead of a pump, participants could have collected the score from the balloon and finished the trial. Ninety balloons were presented in the task. Each successful pump increased the reward score and the probability of the balloon burst. The probability of the balloon burst after the third pump was $1 / 18$, after the fourth pump was $1 / 17$, and so on, until the 20th pump, where the probability of the balloon burst was $1 / 1$. Participants did not have time limit to make their decision after the balloon was presented on the screen. Between each response and feedback stimulus, a 1000-1200 ms random delay was introduced. If the balloon popped, the negative feedback stimulus was presented for $3000 \mathrm{~ms}$, similarly to the screen indicating the successful collection of the score. A new empty balloon appeared on the screen $10 \mathrm{~ms}$ after these events. 

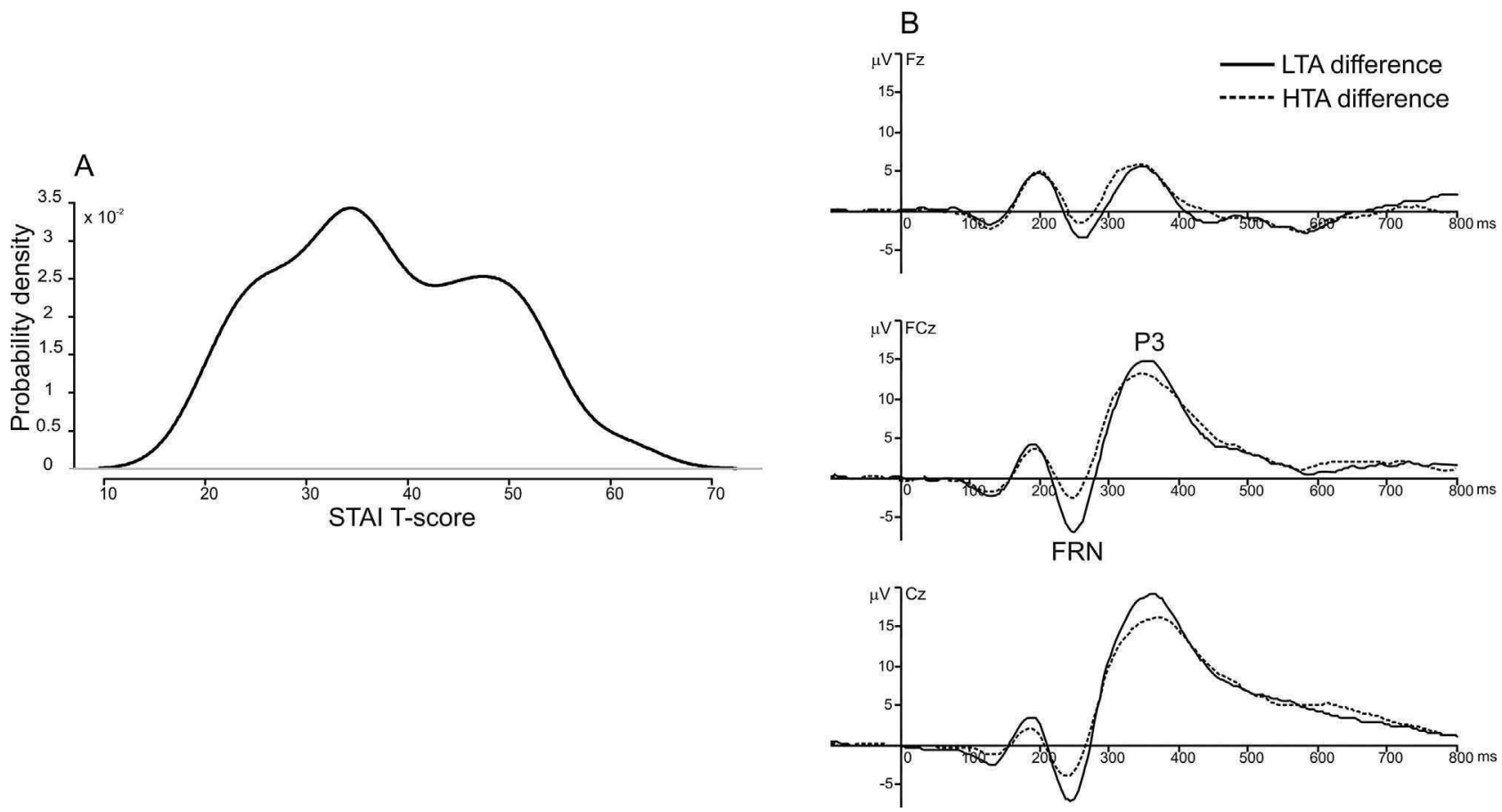

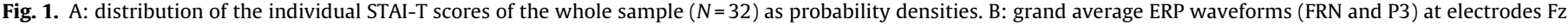
FCz, and Cz for each group. LTA: low trait anxiety, HTA: high trait anxiety. Time zero is the presentation of feedback.

Table 1

Measurements in the two groups: questionnaires and behavioral data.

\begin{tabular}{|c|c|c|c|c|}
\hline & $\begin{array}{l}\operatorname{LTA}(n=13) \\
M(\mathrm{SD})\end{array}$ & $\begin{array}{l}\operatorname{HTA}(n=13) \\
M(\mathrm{SD})\end{array}$ & $t$ value & Cohen's $d$ \\
\hline Age [years] & $21.2(1.1)$ & $21.4(1.7)$ & -0.41 & -0.17 \\
\hline BIS TS & $58.77(11.3)$ & $64.77(11.3)$ & -1.36 & -0.55 \\
\hline BSSS TS & $16.62(4.8)$ & $6.2)$ & -0.18 & -0.07 \\
\hline STAI-T $^{* * *}$ & $28.69(5.3)$ & $46.77(6.7)$ & -7.65 & -3.12 \\
\hline BDI TS $S^{* *}$ & $9.46(0.8)$ & $11.54(2.1)$ & -3.28 & -1.34 \\
\hline Verbal fluency [correct items] & $113.6(17.7)$ & $106.7(17.9)$ & 0.99 & 0.4 \\
\hline Listening span level ${ }^{\mathrm{a}}$ & $3.44(0.9)$ & $3.03(0.6)$ & 1.3 & 0.53 \\
\hline Go/No-Go [discriminability] & $0.29(0.3)$ & $0.33(0.2)$ & -0.45 & -0.18 \\
\hline Mean adjusted pumps & $8.12(1.1)$ & $8.39(1.4)$ & -0.55 & -0.23 \\
\hline Number of balloon bursts & $36.69(6.1)$ & $38(8.6)$ & -0.45 & -0.18 \\
\hline Total score & $2087.7(336.5)$ & $2143.4(349.4)$ & -0.41 & -0.17 \\
\hline
\end{tabular}

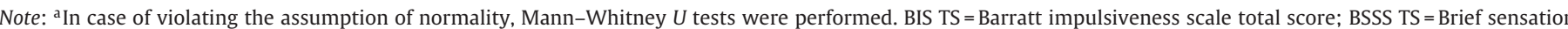
seeking scale total score; STAI-T = T-Anxiety score; BDI TS = Beck depression inventory short version total score.

The behavioral outcome measures of the BART were the mean adjusted number of pumps across balloons (mean number of pumps on balloons that did not explode), the number of balloon bursts, and the total score at the end of the experiment [18]. After the EEG experiment, they performed three neuropsychological tasks assessing EFs [32], which could be impaired in HTA [33] and could be involved in BART performance [19,22]: a verbal fluency task, a listening span task, and a Go/No-Go Task. We did not find group differences in the behavioral measures of the BART or in EFs (see Table 1). There was a significant difference in BDI score between the two groups, $t(24)=-3.28, p<0.05$. With neuropsychological measurements and additional questionnaires we could make sure that our groups differ only in anxiety and general mood state. We could also exclude the effect of EFs on FRN and P3 [22].

\subsection{EEG measurement and analysis}

EEG activity was registered with a 128 channel Geodesic Sensor Net (GES 300; Electrical Geodesics, Inc., Eugene, OR). Electrode $\mathrm{Cz}$ was used as a reference and a sampling rate of $500 \mathrm{~Hz}$ was applied. Before the offline analysis, bad channels were replaced by spline interpolation. A band-pass filter $(0.3-30 \mathrm{~Hz}, 48 \mathrm{~dB} /$ oct $)$ and a $50 \mathrm{~Hz}$ notch filter were applied. To correct eye-movement and heartbeat artifacts, independent component analysis (ICA) was used. EEG data was re-referenced to the average activity of all electrodes.

Epochs extending from -100 to $1000 \mathrm{~ms}$ were time-locked to the presentation of negative (balloon burst) or positive (increasing balloon and score) stimuli. We used the average activity from -100 to $0 \mathrm{~ms}$ as a prestimulus baseline.

An automatic artifact rejection algorithm implemented in Brain Vision Analyzer software (Brain Products $\mathrm{GmbH}$, Munich, Germany) was used with four criteria: the maximum gradient allowed for an epoch was $50 \mu \mathrm{V} / \mathrm{ms}$, segments where the activity exceeded $+/-100 \mu V$ were rejected, the lowest activity allowed was $0.5 \mu \mathrm{V}$, and the maximum absolute difference between the minimum and maximum voltages in an epoch was $200 \mu \mathrm{V}$. Less than 19 artifact-free epochs for negative feedback was an exclusion criterion [21,22]. The average number of the kept segments in the negative feedback condition was $31.2(\mathrm{SD}=7.84$; range: $19-48)$ for the final sample. 
In the next step of the offline analysis, positive and negative stimuli-locked epochs were averaged separately; then, the positive feedback waveform was subtracted from the negative feedback waveform to create a difference wave [21]. Since previous ERP studies with the BART $[15,21,22]$ used frontocentral channels, we chose E11 (identical to $\mathrm{Fz}$ position), E6 (identical to $\mathrm{FCz}$ position) and $\mathrm{Cz}$ for statistical analysis. Moreover, the FRN appeared to be the largest in these positions. We measured the peak amplitude and latency of FRN (time interval: 200-300 ms) and P3 (time interval: 300-600 ms) components. Two-way mixed ANOVAs were used with Group (LTA, HTA) as a between-subjects factor and Electrode (Fz, FCz, Cz) as a within-subjects factor. We used LSD (least significant difference) tests for pair-wise comparisons. The Greenhouse-Geisser epsilon $(\epsilon)$ correction was applied when necessary.

\section{Results}

Grand average ERP difference waves (FRN and P3) for each group are presented in Fig. 1B. A 2 (Group) $\times 3$ (Electrode) ANOVA was conducted on FRN peak amplitude. The main effect of Group was significant, $F(1,24)=11.78, p<0.01, \eta_{p}^{2}=0.33$, and also the main effect of Electrode, $F(2,48)=7.39, \epsilon=0.573, p<0.01, \eta_{\mathrm{p}}{ }^{2}=0.24$. The FRN peak amplitude was smaller (less negative) in the HTA group than in the LTA group $(-3.33 \mu \mathrm{V}$ vs. $-6.96 \mu \mathrm{V})$. The same ANOVA was performed on FRN peak latency data. The main effect of Electrode was significant $F(2,48)=33.83, \epsilon=0.78, p<0.001$, $\eta_{\mathrm{p}}{ }^{2}=0.59$. The Group $\times$ Electrode interaction was also significant, $F(2,48)=4.02, \epsilon=0.78, p<0.05, \eta_{p}{ }^{2}=0.14$. Pair-wise comparisons indicated that in the HTA group, the FRN was more delayed at Fz than at $\mathrm{FCz}(p<0.001)$ or at $\mathrm{Cz}(p<0.001)$. In the LTA group, the FRN was delayed at Fz compared to $\mathrm{Cz}(p<0.001)$. However, differences between HTA and LTA groups in FRN peak latency at each electrode site were not significant.

A 2 (Group) $\times 3$ (Electrode) ANOVA was conducted on P3 peak amplitude, as well. The main effect of Electrode was significant, $F(2,48)=44.63, \epsilon=0.691, p<0.001, \eta_{\mathrm{p}}{ }^{2}=0.65$. The P3 was larger at $\mathrm{FCz}$ than at $\mathrm{Fz}(p<0.001)$, and it was larger at $\mathrm{Cz}$ than at $\mathrm{Fz}$ $(p<0.001)$ or at $\mathrm{FCz}(p<0.001)$. The same ANOVA was performed on P3 peak latency. Only the main effect of Electrode was significant, $F(2,48)=4.08, \epsilon=0.699, p<0.05, \eta_{\mathrm{p}}^{2}=0.15$. The P3 was delayed at $\mathrm{Cz}$ compared to $\mathrm{Fz}(p<0.05)$ or to $\mathrm{FCz}(p<0.01)$.

\section{Discussion}

The aim of this study was to compare feedback processing between LTA and HTA participants in a paradigm that requires a series of risky decisions, and which resembles real-life risk taking. We found attenuated FRN in the HTA group compared to LTA group. The ambiguity presented via opaque gain and loss probabilities in the BART [18] could have triggered differences in task-related processes between HTA and LTA individuals [16]. Namely, HTA participants probably expected more frequent negative feedback after their choices, given their pessimistic expectation bias $[2,8,9]$. Since FRN reflects the prediction error of the outcome [13], it seems that negative events met the expectations of HTA individuals.

Our results are in line with previous studies, in which HTA participants showed smaller FRN after negative feedback [2,9], and larger FRN after ambiguous feedback [9]. These studies used variations of a simple monetary gambling paradigm, where ambiguity was presented at the level of feedback by using positive, negative, and ambiguous outcomes. In the ambiguous condition, participants could win or lose money, but the feedback did not indicate the actual monetary outcome. Therefore, ambiguous outcomes could trigger expectation bias in HTA adults, which means that they interpreted ambiguous outcomes as more threatening than negative outcomes [9]. However, positive and negative conditions did not require processing ambiguous information. While in simple monetary gambling paradigms participants have to take some risk compulsorily $[2,9]$, in the BART, it is possible to collect the reward and leave the risky situation, which resembles frequent real-life circumstances (e.g., "Should I drive over the speed limit?"). This characteristic of the task gives a strong external validity to it $[17,18]$. Effective processing of feedback is crucial when risk becomes too high in a series of action [17].

The effect of emotional factors, such as anxiety and depression, on decision making has been studied using a limited number of paradigms $[7,8,26]$. Studies suggesting the pessimistic expectation bias behind smaller FRN in HTA utilized simple gambling tasks with feedback manipulations [2,9], while other works focusing on the imbalance between emotional and analytic processes in anxiety used variations of the framing effect paradigms [7,8,34]. Another study found attenuated FRN in depressive patients in a simple gambling task [26]. The amplitude of the FRN for positive feedback was also associated with trait anhedonia.

In our study, we provided evidence of smaller FRN in HTA in a gambling task which mimics real-life risk-taking [18]. Moreover, we did not introduce additional feedback manipulation; therefore, our results can stand as general effects of ambiguity on risk-taking decisions in anxiety. As another strength of the study, the groups were matched on BART behavioral performance and did not differ in EFs. This methodological solution helped us to rule out the effects of factors unrelated to anxiety (e.g., general task-solving strategies) in the BART [22]. Moreover, previous studies [2,9] selected HTA and LTA participants on the basis of the lower and upper quartiles of the anxiety scores. In contrast, the present study used post-hoc median split to form groups. While the latter method has limitations as a data driven approach, it also provides a cross-method validation to previous studies as current results are in line with prior ones $[2,9]$.

To establish a framework that can explain neurophysiological findings on pessimistic expectations, emotional hyperactivation, and analytic hypoactivation at the same time, more studies are needed with various decision making paradigms. For future research, studying other aspects of anxiety should be considered, as well. In the present study, we only measured trait anxiety; therefore, we do not have information about state anxiety triggered by the gambling situation. We excluded participants with clinical level of depression. Although this approach makes our interpretation specific to trait anxiety, the characteristics of the sample also limits the generalizability of our results. For future studies, it would be important to compare the effect of different forms of negative affect, such as anhedonia, depression, trait and state anxiety.

\section{Conclusions}

We found evidence for attenuated FRN in HTA adults compared to LTA adults in a risky decision making paradigm. This finding supports the pessimistic expectation bias in anxiety. Since HTA participants expected more frequent balloon bursts, the negative feedback met their biased expectations. Our results suggest that smaller FRN after negative feedback is a general characteristic of HTA and not only triggered by feedback manipulation.

Adaptive functioning in anxiety-related disorders is disturbed by impairments in decision making [5,7,9]. Our finding contributes to the increasing evidence about how anxiety affects processes in decision making and shed light on the related brain mechanisms. To understand the relationship between anxiety and decision making 
would be important not only in clinical but also in neuroeconomical context [7,35].

\section{Acknowledgements}

This research was supported by the Hungarian Scientific Research Fund (OTKA MB08A 84743, OTKA NF 105878) and Janos Bolyai Research Fellowship of the Hungarian Academy of Sciences (to K.J.).

\section{References}

[1] M.W. Eysenck, N. Derakshan, R. Santos, M.G. Calvo, Anxiety and cognitive performance: attentional control theory, Emotion 7 (2007) 336-353, http:// dx.doi.org/10.1037/1528-3542.7.2.336

[2] R. Gu, Y.-X. Huang, Y.-J. Luo, Anxiety and feedback negativity, Psychophysiology 47 (2010) 961-967, http://dx.doi.org/10.1111/j 1469-8986. 2010.00997. x

[3] M. Carrasco, C. Hong, J.K. Nienhuis, S.M. Harbin, K.D. Fitzgerald, W.J. Gehring et al., Increased error-related brain activity in youth with obsessive-compulsive disorder and other anxiety disorders, Neurosci. Lett. 541 (2013) 214-218, http://dx.doi.org/10.1016/j.neulet.2013.02.017

[4] D.M. Olvet, G. Hajcak, The error-related negativity (ERN) and psychopathology: toward an endophenotype, Clin. Psychol. Rev. 28 (2008) 1343-1354, http://dx.doi.org/10.1016/j.cpr.2008.07.003

[5] T. Endrass, S. Koehne, A. Riesel, N. Kathmann, Neural correlates of feedback processing in obsessive-compulsive disorder, J. Abnorm. Psychol 122 (2013) 387-396, http://dx.doi.org/10.1037/a0031496

[6] A.C. Miu, R.M. Heilman, D. Houser, Anxiety impairs decision-making: psychophysiological evidence from an Iowa Gambling Task, Biol. Psychol. 77 (2008) 353-358, http://dx.doi.org/10.1016/j.biopsycho.2007.11.010

[7] C. a Hartley, E. a Phelps, Anxiety and decision-making, Biol. Psychiatry 72 (2012) 113-118, http://dx.doi.org/10.1016/j.biopsych.2011.12.027

[8] M.P. Paulus, A.J. Yu, Emotion and decision-making: affect-driven belief systems in anxiety and depression, Trends Cogn. Sci. 16 (2012) 476-483, http://dx.doi.org/10.1016/j.tics.2012.07.009

[9] R. Gu, Y. Ge, Y. Jiang, Y. Luo, Anxiety and outcome evaluation: the good, the bad and the ambiguous, Biol. Psychol. 85 (2010) 200-206, http://dx.doi.org/ 10.1016/j.biopsycho.2010.07.001

[10] C.B. Holroyd, M.G.H. Coles, The neural basis of human error processing: reinforcement learning, dopamine, and the error-related negativity, Psychol. Rev. 109 (2002) 679-709, http://dx.doi.org/10.1037//0033-295X.109.4.679

[11] D. Talmi, R. Atkinson, W. El-Deredy, The feedback-related negativity signals salience prediction errors, not reward prediction errors, J. Neurosci. 33 (2013) 8264-8269, http://dx.doi.org/10.1523/JNEUROSCI.;1; 5695-12.2013

[12] W.H.R. Miltner, C.H. Braun, M.G.H. Coles, Event-related brain potentials following incorrect feedback in a time-estimation task: evidence for a generic neural system for error detection, J. Cogn. Neurosci. 9 (1997) 788-798, http:// dx.doi.org/10.1162/jocn.1997.9.6.788

[13] C.B. Holroyd, G. Hajcak, J.T. Larsen, The good, the bad and the neutral: electrophysiological responses to feedback stimuli, Brain Res. 1105 (2006) 93-101, http://dx.doi.org/10.1016/j.brainres.2005.12.015

[14] K.R. Ridderinkhof, M. Ullsperger, E. a Crone, S. Nieuwenhuis, The role of the medial frontal cortex in cognitive control, Science 306 (2004) 443-447, http://dx.doi.org/10.1126/science.1100301

[15] A.S. Euser, C.S. van Meel, M. Snelleman, I.H. Franken, Acute effects of alcohol on feedback processing and outcome evaluation during risky decision-making: an ERP study, Psychopharmacology (Berl) 217 (2011) 111-125, http://dx.doi.org/10.1007/s00213-011-2264-x

[16] G.G. Knyazev, A.N. Savostyanov, E. a Levin, Uncertainty, anxiety, and brain oscillations, Neurosci. Lett. 387 (2005) 121-125, http://dx.doi.org/10.1016/j. neulet.2005.06.016

[17] S.M. Helfinstein, T. Schonberg, E. Congdon, K.H. Karlsgodt, J. a. Mumford, F.W Sabb, et al., Predicting risky choices from brain activity patterns, Proc. Natl. Acad. Sci. U S A 111 (2014) 2470-2475, http://dx.doi.org/10.1073/pnas. 1321728111
[18] C.W. Lejuez, J.P. Read, C.W. Kahler, J.B. Richards, S.E. Ramsey, G.L. Stuart, et al., Evaluation of a behavioral measure of risk taking: the balloon analogue risk task (BART), J. Exp. Psychol. Appl. 8 (2002) 75-84, http://dx.doi.org/10.1037// 1076-898X.8.2.75

[19] J.A. Campbell, J.R. Samartgis, S.F. Crowe, Impaired decision making on the balloon analogue risk task as a result of long-term alcohol use, J. Clin. Exp. Neuropsychol. 00 (2013) 1-11, http://dx.doi.org/10.1080/13803395.2013. 856382

[20] S. Fecteau, A. Pascual-Leone, D.H. Zald, P. Liguori, H. Théoret, P.S. Boggio, et al. Activation of prefrontal cortex by transcranial direct current stimulation reduces appetite for risk during ambiguous decision making, J. Neurosci. 27 (2007) 6212-6218, http://dx.doi.org/10.1523/JNEUROSCI.;1; 0314-07.2007

[21] G. Fein, M. Chang, Smaller feedback ERN amplitudes during the BART are associated with a greater family history density of alcohol problems in treatment-naïve alcoholics, Drug Alcohol Depend. 92 (2008) 141-148, http:// dx.doi.org/10.1016/j.drugalcdep.2007.07.017

[22] A. Kóbor, A. Takács, K. Janacsek, D. Németh, F. Honbolygó, V. Csépe, Different strategies underlying uncertain decision making: higher executive performance is associated with enhanced feedback-related negativity, Psychophysiology (2014), http://dx.doi.org/10.1111/psyp.12331

[23] C.D. Spielberger, R.L. Gorsuch, R.E. Lushene, STAI Manual for the State-Trait Anxiety Inventory (Self-Evaluation Questionnaire), Consulting Psychologists Press, Palo Alto, CA, 1970.

[24] K. Sipos, M. Sipos, The development and validation of the Hungarian form of the state-trait anxiety inventory, Ser. Clin. Community Psychol. Stress Anxiety 2 (1983) 27-39.

[25] S. Rózsa, E. Szádóczky, J. Füredi, A Beck depresszió kérdőív rövidített változatának jellemzői hazai mintán [psychometric properties of the Hungarian version of the shortened Beck Depression Inventory] [Hungarian], Psychiatr. Hung. 16 (2001) 384-402.

[26] W. Liu, L. Wang, H. Shang, Y. Shen, Z. Li, E.F.C. Cheung, et al., Feedback negativity and anhedonia depression, Neuropsychologia 53 (2014) 213-220.

[27] J.H. Patton, M.S. Stanford, E.S. Barratt, Factor structure of the Barratt Impulsiveness Scale, J. Clin. Psychol. 51 (1995) 768-774, http://dx.doi.org/10. 1002/1097-4679(199,511) 51:6<768::aid-jclp2270510607>3.0. co;2-1

[28] G. Varga, A. Szekely, P. Antal, P. Sarkozy, Z. Nemoda, Z. Demetrovics, et al., Additive effects of serotonergic and dopaminergic polymorphisms on trait impulsivity, Am. J. Med. Genet. B Neuro Psychiatr. Genet. 159B (2012) 281-288, http://dx.doi.org/10.1002/ajmg.b.32025

[29] R.H. Hoyle, M.T. Stephenson, P. Palmgreen, E.P. Lorch, R.L. Donohew, Reliability and validity of a brief measure of sensation seeking, Pers. Individ. Dif. 32 (2002) 401-414, http://dx.doi.org/10.1016/S0191-8869(01) 32-0

[30] R. Urbán, Smoking outcome expectancies mediate the association between sensation seeking, peer smoking, and smoking among young adolescents, Nicotine Tob. Res. 12 (2010) 59-68, http://dx.doi.org/10.1093/ntr/ntp174

[31] A.T. Beck, C.H. Ward, M. Mendelson, J. Mock, J. Erbaugh, An inventory for measuring depression, Arch. Gen. Psychiatry 4 (1961) 561-571, http://dx.doi. org/10.1001/archpsyc.1961.01710120031004

[32] A. Miyake, N.P. Friedman, M.J. Emerson, A.H. Witzki, A. Howerter, T.D. Wager, The unity and diversity of executive functions and their contributions to complex frontal lobe tasks: a latent variable analysis, Cogn. Psychol. 41 (2000) 49-100, http://dx.doi.org/10.1006/cogp.1999.0734

[33] Y. Fujii, N. Kitagawa, Y. Shimizu, N. Mitsui, A. Toyomaki, N. Hashimoto, et al., Severity of generalized social anxiety disorder correlates with low executive functioning, Neurosci. Lett. 543 (2013) 42-46, http://dx.doi.org/10.1016/j. neulet.2013.02.059

[34] P. Xu, R. Gu, L.S. Broster, R. Wu, N.T. Van Dam, Y. Jiang, et al., Neural basis of emotional decision making in trait anxiety, J. Neurosci. 33 (2013) 18641-18653, http://dx.doi.org/10. 1523/JNEUROSCI.; 1 ; 1253-13.2013

[35] T. Schonberg, C.R. Fox, R. a Poldrack, Mind the gap: bridging economic and naturalistic risk-taking with cognitive neuroscience, Trends Cogn. Sci. 15 (2011) 11-19, http://dx.doi.org/10.1016/j.tics.2010.10.002. 